\title{
Visible Versus Near-Infrared Optical Performance of Diffractive Multifocal Intraocular Lenses
}

\author{
Fidel Vega, María S. Millán, Núria Vila-Terricabras, and Francisco Alba-Bueno \\ Departament d'Òptica i Optometria, Universitat Politècnica de Catalunya, Barcelonatech, Terrassa, Spain
}

Correspondence: Fidel Vega, Departament d'Òptica i Optometria, Universitat Politècnica de Catalunya, Barcelonatech, Violinista Vellsolà 37, 08222 Terrassa, Spain

fvega@oo.upc.edu.

Submitted: July 10, 2015

Accepted: October 6, 2015

Citation: Vega F, Millán MS, VilaTerricabras N, Alba-Bueno F. Visible versus near infrared optical performance of diffractive multifocal intraocular lenses. Invest Ophthalmol Vis Sci. 2015;56:XXX-XXX. DOI:10.1167/ iovs. 15-17664
Purpose. The purpose of this study was to compare the optical performance of diffractive multifocal intraocular lenses (DMIOLs) with visible (VIS) illumination with that of near infrared (NIR) illumination, the latter being used to test pseudophakic eyes in clinical aberrometers and double-pass systems.

Methods. Two DMIOLs of different design (Tecnis $+2.75 \mathrm{D}$ ZKB00 and AcrySof $+2.5 \mathrm{D}$ SV25T0) were tested in vitro in a model eye under both VIS $(\lambda=530 \mathrm{~nm})$ and NIR $(\lambda=780$ $\mathrm{nm}$ ) illumination, and variations in the add power were determined. Moreover, for the two wavelengths, the energy efficiency and modulation transfer function at the DMIOLs' far and near foci were measured with pupils of 3.0 and $4.5 \mathrm{~mm}$. Two counterpart monofocal IOLs (Tecnis ZA9003 and AcrySof SN60WF) were included as references in the comparison.

Results. With VIS light, the two DMIOLs produced relatively well-contrasted images at their near and far foci. Under NIR illumination, the add power increased, whereas the energy efficiency of the near (far) focus decreased (increased). Hence, the DMIOLs tended to behave like monofocal lenses because they generated good quality well-contrasted images only at their far foci.

Conclusions. In addition to changes in add power, the optical performances of the DMIOLs measured under either VIS or NIR illumination are considerably different. Whereas they show two distinct (near and far) foci under VIS light, their optical performances under NIR illumination are clearly biased in favor of their far focus. These results may help prevent a misleading use of NIR-based clinical instruments for the assessment of eyes implanted with DMIOLs.

Keywords: aberrometers, cataract surgery, diffractive multifocal intraocular lens, intraocular lens, pseudophakic eye, visual function testing
$\mathbf{R}$ emoval of the crystalline lens followed by implantation of a multifocal intraocular lens (MIOL) is presently a surgical procedure primarily aimed at providing pseudoaccommodation to patients after cataract surgery. Diffractive MIOLs (DMIOLs) have proven to provide reliable and better clinical outcomes than their refractive MIOL counterparts or accommodating IOLs, ${ }^{1}$ so there is a growing interest in assessing their optical performance in vivo. To objectively determine the imaging quality of DMIOLs in patients, several authors have recently used both wavefront aberrometers ${ }^{2,3}$ and double-pass-based systems. ${ }^{4-6}$ To avoid dazzling the patient, these instruments measure with illuminating wavelengths of the near infrared (NIR) spectral band, typically ranging between 780 and 850 $\mathrm{nm}$. In normal and pseudophakic eyes with monofocal IOLs, ${ }^{7}$ aberrometers are able to correctly account for the change of optical properties between measuring NIR and visible (VIS) light $^{8}$ and thus provide both the objective refraction ${ }^{9}$ and the higher order aberrations of patients. ${ }^{10}$ Moreover, López-Gil and Artal $^{11}$ showed in normal eyes analyzed with the double-pass method that using the central core in the double-pass images and ignoring the tails, the NIR data obtained could be reliably used to predict the modulation transfer function (MTF) of the eye with green light $(543 \mathrm{~nm})$. However, when dealing with pseudophakic eyes implanted with diffractive MIOLs, the simultaneous presence of two or more foci and the strong

Copyright 2015 The Association for Research in Vision and Ophthalmology, Inc iovs.arvojournals.org | ISSN: 1552-5783 dependence that the optical performance of the foci generated from non-zero diffractive orders have on wavelength ${ }^{12,13}$ must be taken into account.

Because the wavelengths of NIR testing are quite different from the wavelengths corresponding to the maximum value of the photopic sensitivity of the eye under visible (VIS) spectrum (approximately $550 \mathrm{~nm}$ ), for which DMIOLs are designed, there is a mismatch between the measured (NIR) and (VIS) wavelengths, which gives rise to the following issues:

- A change in add power of the DMIOL between VIS and NIR (the larger the NIR wavelength, the larger the measured add value), ${ }^{12}$ which in turn, has an effect on halo formation and size. ${ }^{14,15}$

- A change in distribution of energy between the foci of the DMIOL. ${ }^{16}$ This variation in the energetic balance between the distant and near foci is related to the dependence of the diffraction efficiency with wavelength. ${ }^{13}$

- A significant difference in the optical quality of the DMIOLs' foci is dependent on the illumination wavelength. ${ }^{12,13}$ This difference is produced by the joint combination of the two former effects.

These phenomena are usually described in the context of wavefront optics, ${ }^{17}$ but their origin and implications in clinical practice can be elusive for specialists who are not familiar with

. 
the principles of diffraction optics. In this sense, the use of NIR-based techniques to in vivo characterize the optical performance of DMIOLs may lead to incorrect conclusions, as pointed out previously. ${ }^{18}$

In vitro optical bench testing of MIOLs ${ }^{19,20}$ is complementary to clinical assessments because it is objective and independent of the patient. Moreover, testing of the lens performance with different wavelengths is straightforward. In this paper we present a new in vitro comparison between the imaging quality of two DMIOLs of different design, the fullaperture Tecnis ZKB00 (AMO Groningen, Groningen, The Netherlands) and the apodized AcrySof ReSTOR SV25T0 (Alcon, Fort Worth, TX, USA), when they are tested on an optical bench under VIS and NIR illumination. By means of a simple yet rigorous image analysis procedure, we show the large differences in energy distribution between far and near images as a function of wavelength (VIS vs. NIR). The implications that such differences have on MTF are discussed by comparing the results obtained with the two DMIOLs. Moreover, the study includes the results obtained with the monofocal counterpart IOLs (Tecnis ZA9003 and AcrySof SN60WF).

\section{METHODS \\ DMIOL Characteristics}

Two bifocal DMIOLs and two monofocal IOLs were studied. The Tecnis ZKB00 DMIOL, with +2.75 diopter (D) add power, has an aspheric anterior surface. The diffractive profile covers the full aperture of the lens and consists of 15 diffractive rings with step boundaries of the same height, intended for approximately equal light distribution between the far and near foci, independent of pupil size. The wavefront-designed aspheric optics of this DMIOL produce a maximum spherical aberration (SA) of $-0.27 \mu \mathrm{m}$ for a $6.0-\mathrm{mm}$ pupil.

On the other hand, the AcrySof ReSTOR SV25T0 DMIOL with $+2.5 \mathrm{D}$ add power has an anterior apodized diffractive surface (3.4-mm diameter) within which there is a central refractive zone $(1.0 \mathrm{~mm}$ diameter approximately). The outer region of the lens to the 6-mm edge is purely refractive, and thus, the central and outer refractive parts of the lens are intended for distance vision. In addition, the diffractive area presents seven concentric rings with step boundaries of decreasing height, which allows for an asymmetrical and pupil-dependent light distribution between the far and near foci that benefits the far focus for large pupils. ${ }^{21}$ The anterior surface of the SV25T0 is designed with negative SA of -0.20 $\mu \mathrm{m}$ for a $6.0-\mathrm{mm}$ pupil.

Additionally, the reference monofocal Tecnis ZA9003 (AMO Groningen) and AcrySof SN60WF (Alcon Laboratories) IOLs were included in our study. We emphasize that each monofocal IOL shares the same aspherical design and the same material, with its diffractive counterpart. Thus, the lenses of each pair (DMIOL and monofocal counterpart) have similar characteristics regarding the compensation for high order aberration (mainly SA) and the spectral variation of the refractive index.

All studied lenses had a base optical power of $20 \mathrm{D}$, which in the case of the DMIOLs corresponded to distance focus.

\section{Experimental Setup for Optical Imaging Quality Assessment}

Assessment of the optical imaging quality of the IOLs was made using an optical test bench with a model eye (artificial cornea plus wet cell) that has been described in detail elsewhere. ${ }^{15,22,23}$ Supplementary Figure S1 shows a sketch of the setup, which was in agreement with International Standard Organization (ISO) 11979-2 and 11979-9 recommendations, ${ }^{24,25}$ except for the artificial cornea that was not an aberration-free doublet because this was not representative of the average human cornea, which has intrinsic positive SA. ${ }^{26,27}$ Instead, we used a double convex lens that provided a level of SA at the IOL plane of $+0.27 \mu \mathrm{m}$ for a $6.0-\mathrm{mm}$ pupil, ${ }^{28}$ similar to the one induced by the human cornea on average. ${ }^{29}$ Additionally, it showed a dependence of SA on pupil size close to that of physiological eye models, namely the Liou-Brenan and Holladay models. ${ }^{22,23}$

Light sources consisted of green and NIR light-emitting diodes (LEDs; Thorlabs GmbH, Munich, Germany) with emissions centered at $530 \mathrm{~nm}$ and $780 \mathrm{~nm}$, respectively. Both sources had a full-width half-maximum spectral band width of $32 \mathrm{~nm}$, which represents $6 \%$ (at $530 \mathrm{~nm}$ ) and $4 \%$ (at $780 \mathrm{~nm}$ ) of the central emission line of the LEDs. Either the green or NIR LED illuminated the test object located at the front focal plane of a collimator (200 $\mathrm{mm}$ focal length). Test objects were a 200$\mu \mathrm{m}$ pinhole, for assessment of energy distribution at the foci planes (far and near), and a four-slit pattern for MTF measurements. ${ }^{15}$ The collimated beam illuminated the model eye with the IOL under test, and an iris diaphragm was used to control pupil diameter. All pupil diameters mentioned in this work are referred to in the IOL plane. ${ }^{15,23}$

Inserted in the wet cell of the model eye, the studied DMIOL simultaneously formed two (far and near) images of the test object. These images were magnified and focused onto an 8-bit charge-coupled device (CCD) camera (Wells Research, Inc., West Covina, CA, USA) by means of a $10 \times$ infinite corrected microscope (Wells Research, Inc., West Covina, CA, USA) mounted on a high-precision translation holder. It was checked for both wavelengths that the CCD sensor response was linear in the dynamic range of interest. To improve signalto-noise ratio, each image was eventually the result of averaging eight image frames. A similar procedure was carried out with the monofocal IOLs. Images recorded with green and NIR illumination are referred to hereafter as VIS and NIR images, respectively.

\section{Energy Assessment and MTF Measurements by Image Analysis}

The method of characterizing the energy distribution at the focal planes of a DMIOL has been reported in detail elsewhere $e^{22,23}$ and is illustrated in Figure 1. The figure shows the VIS image of the pinhole object captured at the far focus of the ZKB00 DMIOL. Although the image in Figure 1 is shown in logarithmic scale of intensity, for the sake of visualization, ${ }^{15,30}$ the original intensity values in the linear scale provided by the digital 8-bit CCD camera were used in the calculations described below.

Briefly, the image in a given focal plane consisted of the cores harp image of the pinhole object surrounded by a blurred halo-shaped background. This background is principally formed from an out-of-focus image produced by the other focus of the MIOL but may have additional contributions from a variety of factors such as the energy expended in higher diffraction orders, ${ }^{13}$ scattering produced by the diffractive steps of the lens, ${ }^{31}$ and residual level of higher order aberrations, mainly related to insufficient correction of the corneal SA. ${ }^{22,23}$ Because the gray level of a pixel of the image is proportional to the energy impinging on that pixel, it is possible to compute the energy of the light that reaches a particular region of the image by integrating the gray level of all pixels belonging to that region. In this way, the energy of the total image ( $E_{\text {total }}$ ), which consists of the core (sharp image of the pinhole) plus the background regions and the energy just 


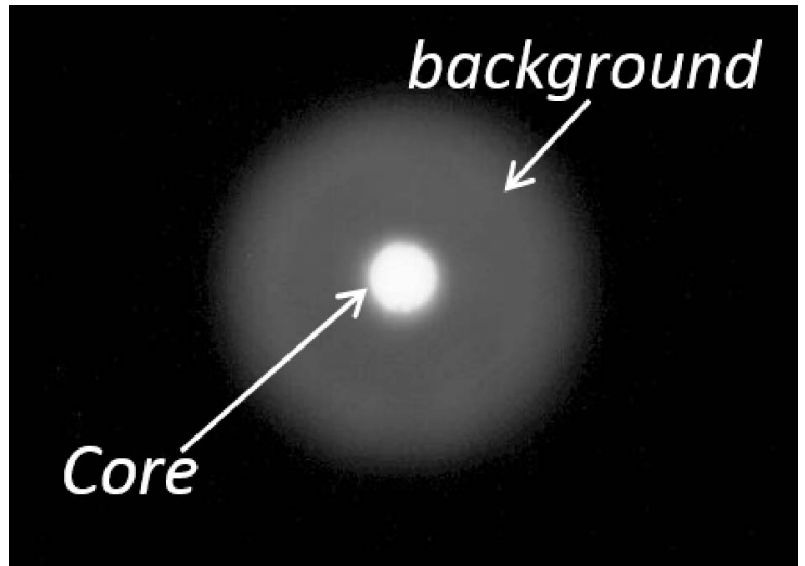

FIGURE 1. Image of the pinhole object in logarithmic scale of intensity obtained with green (VIS) illumination at the far focus of the ZKB00 DMIOL. Arrows indicate regions named core and background, respectively.

in the focused pinhole region $\left(E_{\text {core }}\right)$, were computed separately, The energy in the background $\left(E_{\text {backg }}\right)$ could be calculated from the difference, $\left(E_{\text {total }}\right)-\left(E_{\text {core }}\right)$. Finally, the normalized energy ( $\left.N E_{\text {core }}\right)$, which corresponds to the percentage of energy correctly focused in the core region, and $\left(N E_{\text {backg }}\right)$, the percentage of the energy spread in the background, were obtained as the ratios:

$$
\left(N E_{\text {core }}\right)=\frac{\left(E_{\text {core }}\right)}{\left(E_{\text {total }}\right)} \times 100
$$

and

$$
\left(N E_{\text {backg }}\right)=\frac{\left(E_{\text {backg }}\right)}{\left(E_{\text {total }}\right)} \times 100
$$

The procedure outlined above was also followed in the case of NIR images.

As for MTF curves, they were obtained as described previously, ${ }^{15,32}$ using the corresponding VIS and NIR images of the four slit pattern and calculating the Fourier transform of the line spread function. In our setup, the spatial frequency of 50 cycles/mm approximately corresponded to an angular spatial frequency of 30 cycles per degree. The criterion for determining the best planes of focus was to choose those that maximized the area under the MTF curves. The MTFs for both VIS and NIR illumination for all the IOLs were obtained for pupil sizes of 3.0 and $4.5 \mathrm{~mm}$.

\section{Results}

Figure 2 shows the VIS (Figs. 2a, 2b) and NIR (Figs. 2c, 2d) images of the pinhole object obtained with a $4.5-\mathrm{mm}$ pupil at the near (Figs. 2a, 2c) and far (Figs. 2b, 2d) foci of the fullaperture ZKB00 DMIOL. The VIS and NIR images obtained with the counterpart monofocal ZA9003 are shown in Figures $2 \mathrm{e}, 2 \mathrm{f}$, respectively. Figure 2 includes the percentage of the energy correctly focused $\left(N E_{\text {core }}\right)$ in each foci. The values of the VIS and NIR core and background energies obtained with pupil sizes of 3.0 and $4.5 \mathrm{~mm}$ are summarized in Table 1 .

Starting with results obtained with VIS light, and for both pupils, it can be seen that the ZKB00 DMIOL splits the energy between its near and far foci in a balanced way because $\left[\left(N E_{\text {core }}\right)_{n e a r}\right]^{\mathrm{VIS}}$ and $\left[\left(N E_{\text {core }}\right)_{\text {far }}\right]^{\mathrm{VIS}}$ are similar, with values on the order of $40 \%$, although there is a slight reduction of these values for the largest pupil. Consequently, the background energies in the near and far foci $\left(\left[\left(N E_{\text {backg }}\right)_{\text {near }}\right]^{\mathrm{VIS}}\right.$ and $\left.\left[\left(N E_{\text {backg }}\right)_{\text {far }}\right]^{\mathrm{VIS}}\right)$ are also similar, with values of approximately $60 \%$,which tend to slightly increase with pupil.

Remarkably, in the case of NIR images, the energy distribution between the two foci is no longer balanced but, on the contrary, different values of the energy correctly focused in the near and far foci are found. In particular, there is a large reduction in $\left[\left(N E_{\text {core }}\right)_{\text {near }}\right]^{\mathrm{NIR}}$ and a large increase in $\left[\left(N E_{\text {core }}\right)_{f a r}\right]^{\mathrm{NIR}}$ in comparison to those of the VIS images. For instance, with NIR illumination and a $4.5-\mathrm{mm}$ pupil, $\left[\left(N E_{\text {core }}\right)_{\text {near }}\right]^{\mathrm{NIR}}=22 \%$, whereas $\left[\left(N E_{\text {core }}\right)_{\text {far }}\right]^{\mathrm{NIR}}=70 \%$. The opposite occurs for the background energies.

The increase in add power of the lens as a result of the change from VIS to NIR illumination is also shown in Figure 2 through the halo size. In the near focus of the ZKB00
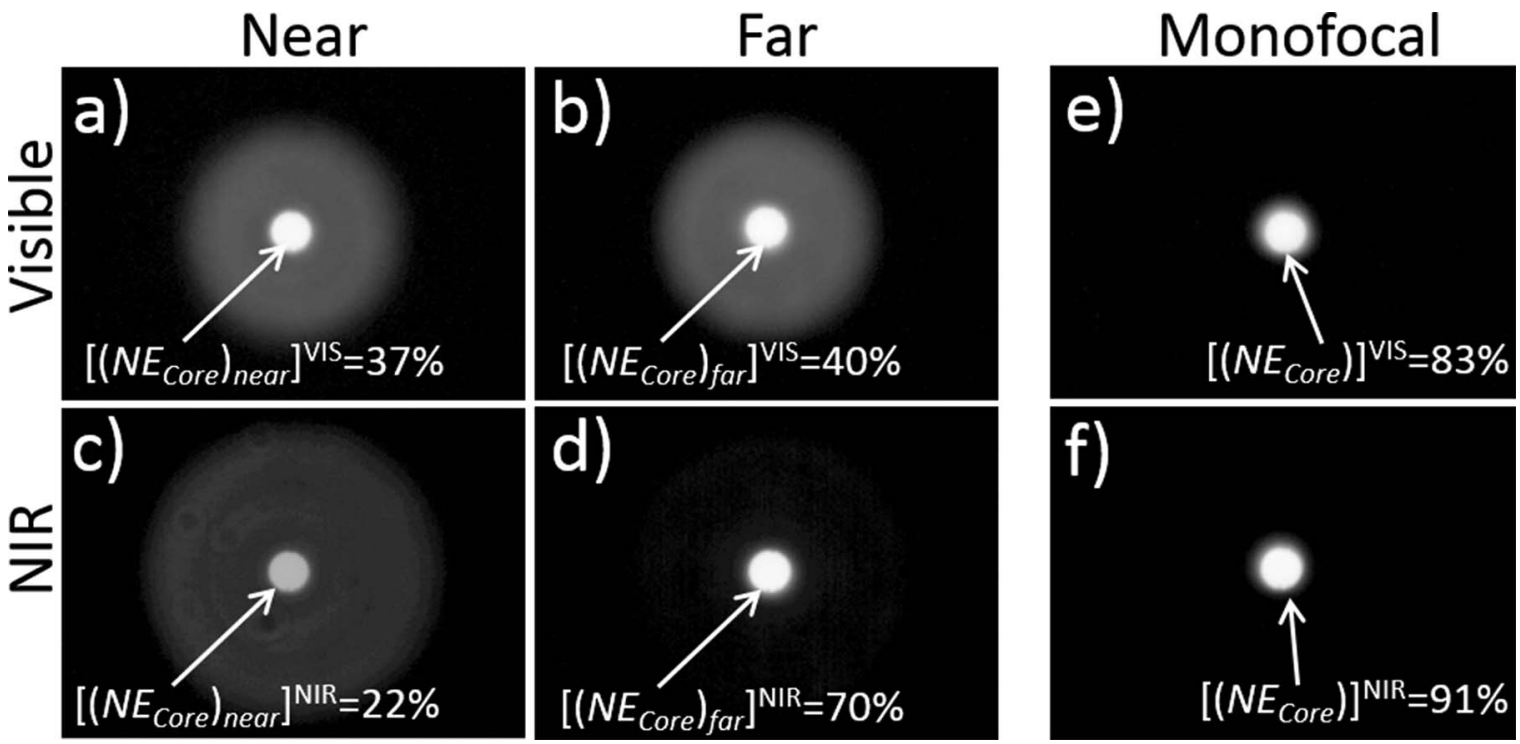

Figure 2. Images of the pinhole object in logarithmic scale of intensity obtained at the near and far foci of the ZKBOO DMIOL (a, b, c, d) and at the focus of the monofocal ZA9003 IOL $(\mathbf{e}, \mathbf{f})$ under VIS $(\mathbf{a}, \mathbf{b}, \mathbf{e})$ and NIR $(\mathbf{c}, \mathbf{d}, \mathbf{f})$ illumination. $\left[(N E)_{\text {core }}\right]^{\mathrm{VIS}}$ and $\left[(N E)_{\text {core }}\right]^{\mathrm{NIR}}=$ percentage of energy at the core region with VIS and NIR illumination. 
TABLe 1. Normalized Core and Background Energy Levels at the Foci of ZKBOO DMIOL

\begin{tabular}{lcccccc}
\hline \multirow{2}{*}{$\begin{array}{l}\text { Pupil Size, } \\
\text { mm }\end{array}$} & & \multicolumn{2}{c}{ Near Foci } & & \multicolumn{2}{c}{ Far Foci } \\
\cline { 3 - 4 } & $\lambda, \mathbf{n m}$ & $\mathbf{N E}_{\text {core }}$ & $\mathbf{N E}_{\text {backg }}$ & & $\mathbf{N E}_{\text {core }}$ & NE $_{\text {backg }}$ \\
\hline 3.0 & 530 & $41 \pm 1$ & $59 \pm 1$ & & $44 \pm 1$ & $56 \pm 1$ \\
& 780 & $25 \pm 2$ & $75 \pm 6$ & & $71 \pm 5$ & $29 \pm 2$ \\
4.5 & 530 & $37 \pm 1$ & $63 \pm 1$ & & $40 \pm 1$ & $60 \pm 1$ \\
& 780 & $22 \pm 2$ & $78 \pm 5$ & & $70 \pm 4$ & $30 \pm 2$
\end{tabular}

$\mathrm{NE}_{\mathrm{backg}}$, percentage of the energy spread in the background; $\mathrm{NE}_{\mathrm{core}}$, percentage of the energy correctly focused in the core region.

Table shows normalized percentages of core and background energy levels calculated using Equations 1 and 2 at the foci of the ZKBO0 diffractive multifocal intraocular lenses (DMIOL).

DMIOL, a bigger halo is obtained with NIR than with VIS light. A larger add power in the case of NIR illumination makes the far and near focal planes more distant and, consequently, the defocused image that forms the NIR halo bigger. In addition, its energy has also increased from $\left[\left(N E_{\text {backg }}\right)_{\text {near }}\right]^{\mathrm{VIS}}=63 \%$ up to $\left[\left(N E_{\text {backg }}\right)_{\text {near }}\right]^{\mathrm{NIR}}=78 \%$ (Table 1). This effect, although present in the far focus too, is hardly noticeable due to the halved energy of the big NIR halo in this focus $\left(\left[\left(N E_{\text {backg }}\right)_{f a r}\right]^{\mathrm{NIR}}=30 \%\right)$, with respect to the VIS light $\left(\left[\left(N E_{\text {backg }}\right)_{f a r}\right]^{\mathrm{VIS}}=60 \%\right)$.

In the case of the apodized SV25T0 DMIOL and its monofocal SN60WF counterpart, the VIS and NIR images obtained with a pupil size of $4.5 \mathrm{~mm}$ are shown in Figure 3. In contrast to the ZKB00 DMIOL, the VIS light images formed by the apodized SV25T0 DMIOL (Figs. 3a, 3b) show a biased energy distribution that benefits the far focus because $\left[\left(N E_{\text {core }}\right)_{f a r}\right]^{\mathrm{VIS}}=61 \%$ is nearly five times larger than $\left[\left(N E_{\text {core }}\right)_{\text {near }}\right]^{\mathrm{VIS}}=13 \%$. This behavior becomes even more significant when the NIR light image is used (Figs. 3c, 3d) in order to obtain a faint image of the pinhole at the near focus (Fig. 3c), which is barely discernible from the background and has a $\left[\left(N E_{\text {core }}\right)_{n e a r}\right]^{\mathrm{NIR}}$ of only $6 \%$ of the total energy. Conversely, in the far focus (Fig. 3d), the percentage of energy correctly focused is high with $\left[\left(N E_{\text {core }}\right)_{f a r}\right]^{\mathrm{NIR}}=77 \%$, whereas the one spread in the background is relatively small, with
TABLE 2. Normalized Core and Background Energy Levels at the Foci of SV25T0 DMIOL

\begin{tabular}{|c|c|c|c|c|c|}
\hline \multirow{2}{*}{$\begin{array}{l}\text { Pupil Size, } \\
\text { mm }\end{array}$} & \multirow[b]{2}{*}{$\lambda, \mathbf{n m}$} & \multicolumn{2}{|c|}{ Near Foci } & \multicolumn{2}{|c|}{ Far Foci } \\
\hline & & $\mathrm{NE}_{\text {core }}$ & NE $_{\text {backg }}$ & $\mathbf{N E}_{\text {core }}$ & NE $_{\text {backg }}$ \\
\hline \multirow[t]{2}{*}{3.0} & 530 & $32 \pm 1$ & $68 \pm 2$ & $63 \pm 2$ & $37 \pm 1$ \\
\hline & 780 & $15 \pm 2$ & $85 \pm 6$ & $81 \pm 6$ & $19 \pm 2$ \\
\hline \multirow[t]{2}{*}{4.5} & 530 & $13 \pm 1$ & $87 \pm 1$ & $61 \pm 1$ & $39 \pm 1$ \\
\hline & 780 & $6 \pm 1$ & $94 \pm 5$ & $77 \pm 4$ & $23 \pm 1$ \\
\hline
\end{tabular}

Abbreviations are as shown in Table 1.

Table shows normalized percentages of core and background energy levels calculated using Equations 1 and 2 at the foci of the SV25T0 diffractive multifocal intraocular lenses (DMIOL).

$\left[\left(N E_{\text {backg }}\right)_{f a r}\right]^{\mathrm{NIR}}=23 \%$ and, thus, a well contrasted image of the pinhole can be observed. Table 2 summarizes the results obtained with the SV25T0 DMIOL for both wavelengths and pupil sizes.

Finally, it is worth pointing out again the size of the haloshaped background in the near NIR light image (Fig. 3c) is larger than that in the VIS light image (Fig. 3a). As in the former case of the ZKBOO DMIOL, the larger add power of the DMIOL and the increase in energy that deviated toward the far focus under NIR illumination can explain this intensity distribution.

The MTF curves of the two DMIOLs with both VIS and NIR illumination are shown in Figure 4 for the case of a pupil size of $4.5 \mathrm{~mm}$. Similar results were obtained with a $3.0-\mathrm{mm}$ pupil. The MTF of the corresponding counterpart monofocal IOL is included in these plots for the sake of comparison. It is worth remarking that the MTF curves of the monofocal IOLs must be compared to the ones computed for the far foci of the DMIOLs. We recall that the larger the MTF values, the better the optical quality of the lens.

Starting with the results obtained with VIS light, Figure 4 shows that the MTFs of the monofocal IOLs (SN60WF and ZA9003) reach higher values than the far focus MTFs of their DMIOL counterparts (SV25T0 and ZKB00, respectively) for all the spatial frequencies, although in the case of the SV25T0 DMIOL, the values are close to the ones of the monofocal SN60WF. Another interesting result is that, although the MTF
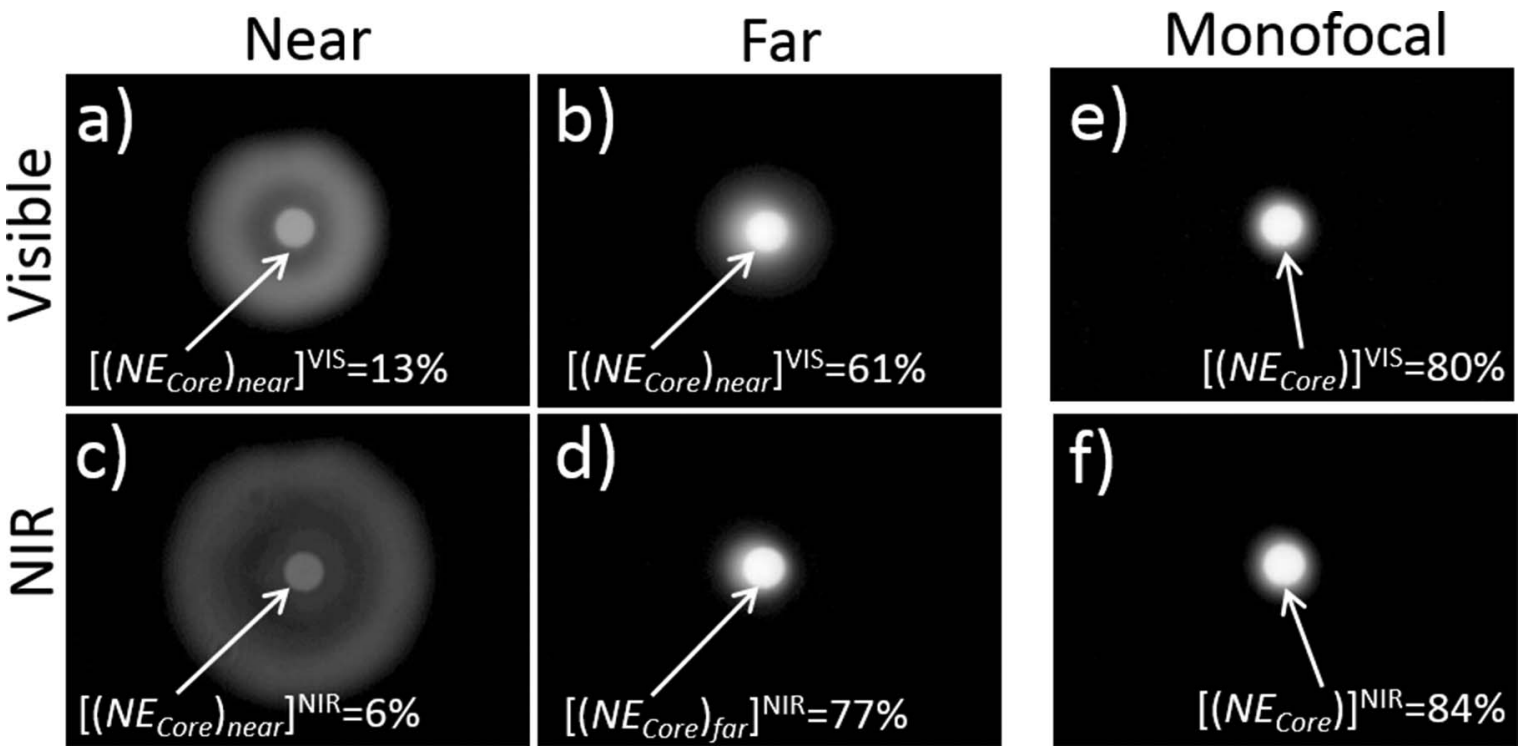

Figure 3. Images of the pinhole object in logarithmic scale of intensity obtained at the near and far foci of the SV25T0 DMIOL (a, b, c, d) and at the focus of the monofocal SN60WF IOL $(\mathbf{e}, \mathbf{f})$ under VIS $(\mathbf{a}, \mathbf{b}, \mathbf{e})$ and NIR $(\mathbf{c}, \mathbf{d}, \mathbf{f})$ illumination. $\left[(N E)_{\text {core }}\right]^{\mathrm{VIS}}$ and $\left[(N E)_{\text {core }}\right]^{\mathrm{NIR}}=$ percentage of energy at the core region with VIS and NIR illumination. 


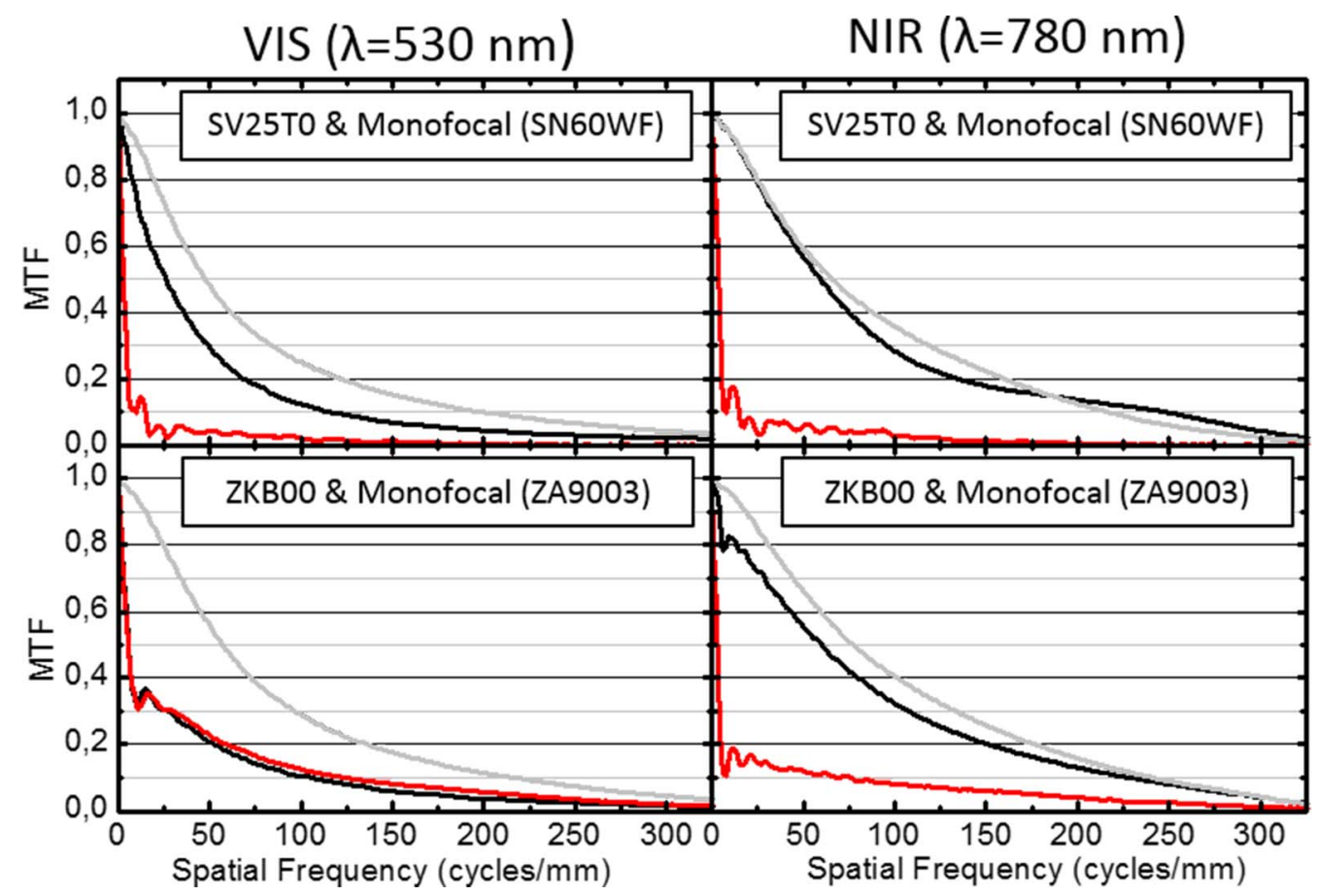

Figure 4. MTF curves were obtained with $4.5-\mathrm{mm}$ pupil under VIS $(\lambda=530 \mathrm{~nm})$ and NIR $(\lambda=780 \mathrm{~nm})$ illumination at the far (black line $)$ and near (red line) foci of the (SV25T0 and ZKB00) DMIOLs and at the focus (gray line) of the monofocal (SN60WF and ZA9003) IOLs.

curves obtained in the near and far foci of the ZKBOO MIOL under VIS light are almost equal, and so must be the optical quality of these foci, there is a difference between the MTFs in the near and far foci of the apodized SV25T0 DMIOL. The latter has a far focus MTF that is considerably higher than the MTF of the near focus for all the spatial frequencies, and therefore, a better optical quality can be expected for the far focus of the apodized SV25T0 DMIOL than for the near one.

Interestingly, with NIR illumination, the MTFs computed at the far focus of both DMIOLs get closer to the MTFs of their monofocal counterparts, especially in the case of the SV25T0, whose MTF practically overlaps the monofocal SN60WFs. Thus, the optical performance at the far foci of the two DMIOLs approaches that of the monofocal IOLs, and overall, there is an improvement in its optical quality with respect to their performance under VIS light. This result is consistent with the similar appearance of Fig. 2d (ZKB00 DMIOL) and Figure 2f (monofocal ZA9003) and Figure 3d (SV25T0 DMIOL) and Figure $3 \mathrm{f}$ (monofocal SN60WF). This similarity, however, cannot be acknowledged when using VIS light (e.g., Figs $2 b$, $2 \mathrm{e}$, or Figs, 3b, 3e).

In addition to the variations concerning the far focus, the MTFs at the near focus of the DMIOLs also change markedly from VIS to NIR light (Fig. 4). Thus, the MTF at the near focus of the ZKBOO DMIOL appears to be lower, and then the optical quality at this focus worsens under NIR light. In the case of the SV25T0 DMIOL, because the optical quality of its near focus was originally low, the deleterious effect produced by changing the testing illumination (VIS for NIR) on the near focus cannot be acknowledged to the same extent.

\section{Discussion}

Images of the pinhole object obtained in the near and far foci of the two DMIOLs (ZKB00 and SV25T0) have proven that there is always a blurred halo-shaped background surrounding the focused image (Figs. 2, 3). With VIS light, the percentage of energy correctly focused on each focus, that is, $\left[\left(N E_{\text {core }}\right)_{\text {near }}\right]^{\text {VIS }}$ and $\left[\left(N E_{\text {core }}\right)_{\text {far }}\right]^{\mathrm{VIS}}$, depends on the particular design of the DMIOL. For instance, the SV25T0 DMIOL has the highest $\left[\left(N E_{\text {core }}\right)_{\text {far }}\right]^{\mathrm{VIS}}$ values for the far focus and the lowest $\left[\left(N E_{\text {core }}\right)_{\text {near }}\right]^{\mathrm{VIS}}$ values for the near focus. This asymmetrical energy distribution tends to be even more biased to benefit the far focus for larger pupils (Table 2 experimental data), which is in agreement with the reported characteristics of the apodized diffractive profile design of this lens. ${ }^{21}$ On the other hand, the ZKBOO DMIOL, with a diffractive profile formed by steps of the same height, shows a balanced distribution of the energy between the two foci, with approximately $40 \%$ of the energy correctly focused in each focus independently of the pupil size (see experimental data of Table 1). Our experimental results are in excellent agreement with the simulated values of VIS diffraction efficiency obtained for this type of lens. ${ }^{12,13}$

More importantly, with NIR illumination, both DMIOLs have shown, on the one hand, a considerable increase in the percentage of energy correctly focused on the far focus, $\left[\left(N E_{\text {core }}\right)_{\text {far }}\right]^{\mathrm{NIR}}$, with an associated improvement in image contrast, and on the other hand, the opposite effect on the near focus, that is, a significant reduction of $\left[\left(N E_{\text {core }}\right)_{n e a r}\right]^{\mathrm{NIR}}$ and worse image contrast. Closely related, in the far focus, the out-of-focus blur associated with the energy of the near focus (which is notorious with VIS light [Figs. $2 \mathrm{~b}$ or $3 \mathrm{~b}$ ]) is strongly reduced and thus, the far NIR images of the DMIOLs resemble those obtained with monofocal IOLs. The consequence, as confirmed by the NIR MTF curves of Figure 4 , is an improvement in the optical quality of the far focus of the DMIOLs, which shows an optical performance fairly close to their monofocal counterparts. Because the opposite occurs with the near focus, one may describe the effect of testing DMIOLs with NIR light, in terms of optical performance, as if they tended to behave like monofocal lenses. This effect is 
even more prominent in the case of apodized DMIOLs with large pupils.

Although our results were experimentally obtained using an optical bench, they may be helpful to correctly interpret clinical outcomes obtained with instruments that use NIR illumination for the assessment of visual function. For instance, visual acuity (VA) at different levels of defocus are commonly obtained with standardized optotype charts using VIS light. In the case of patients with DMIOLs implants, they typically show "bifocal" defocus VA curves (meaning curves with two peaks of VA for far and near distance). ${ }^{2,33}$ However, when an objective pseudoaccommodation analysis was carried out with a double-pass system working at the NIR wavelength of 780 $\mathrm{nm},{ }^{5}$ they showed only "monofocal" defocus curves (i.e., curves with only one peak of far VA), particularly in the case of patients with large pupils and apodized DMIOLs. Closely related, double-pass-based measurements performed in this type of patient with the aim of objectively determining the optical quality of their far vision ${ }^{34}$ would more likely lead to overoptimistic results because, as we have shown, the NIR light images formed by the DMIOLs in their far focus have better contrast and optical quality than the corresponding VIS images (compare Figs. 2b with $3 \mathrm{~b}$ and Figs. 2d with $3 \mathrm{~d}$ ). Conversely, the optical quality of the near focus of such patients would be underestimated in comparison with measurements obtained with VIS light.

A related issue raised by different authors $35,36,37$ arises when measuring patients' wave aberration and, eventually, when deriving some associated metrics such as the MTF from these measurements. ${ }^{2}$ The NIR wavelength used in most commercial aberrometers also dims the near focus as shown in our results. Moreover, the longer the NIR wavelength the weaker the near focus, ${ }^{35,36,37}$ and thus, reported wavefront measurements performed with aberrometers that use longer wavelengths $(808 \text { and } 850 \mathrm{~nm})^{38,33,39}$ in patients who have received DMIOLs implants would produce even more biased results, which would likely be missing the properties associated with the near focus and would correspond only to a benefitted far focus.

Finally, our results have shown another consequence of testing the DMIOLs with NIR: a notorious increase in the size of the halo-shaped background. This effect becomes more evident in the DMIOLs' near NIR light images (Figs. 2c, 3c) because in this plane the background is due to the out-of-focus contribution of the far focus, whose percentage of energy has been increased under NIR illumination. This result is certainly not unexpected because it has been shown that the halo diameter depends on, among other factors, the add power of the DMIOL ${ }^{14,40}$ which turns out to linearly increase with wavelength. ${ }^{12,13,41}$ Therefore, the $+2.75 \mathrm{D}$ and $+2.50 \mathrm{D}$ add powers of the ZKB00 and SV25T0 DMIOLs at $\lambda$ of $550 \mathrm{~nm}$ (VIS light), become $+3.90 \mathrm{D}$ and $3.54 \mathrm{D}$, respectively, under $\lambda$ of 780 $\mathrm{nm}$ (NIR) illumination.

We recall our results were obtained from on-axis analyses, that is, with the MIOLs aligned with the optical system. Earlier works ${ }^{42,43}$ have shown that IOL tilt and/or decentration has an impact on their optical performance. In addition the human eye naturally includes pupil decentration (in respect to the cornea and crystalline lens) as well as lens tilt.

Another potential issue of the study concerns the amount of SA of the artificial cornea. Although there is a general consensus about the need for using artificial cornea models with positive SA to properly test IOLs of aspheric design, ${ }^{29}$ there is not yet an agreement about the specific value of the corneal SA that should be used. For instance, Pieh et al. ${ }^{42}$ used three corneas with SA (6-mm pupil) of $+0.054 \mu \mathrm{m},+0.172 \mu \mathrm{m}$, and $+0.416 \mu \mathrm{m}$, respectively, for in vitro testing of monofocal IOLs. The model cornea described by Carson et al. ${ }^{44}$ had a SA (6-mm pupil) of $+0.2 \mu \mathrm{m}$ and was used to test the SV25T0 DMIOL. The artificial cornea used in our eye model was designed with an SA of $+0,27 \mu \mathrm{m}$ for a 6-mm pupil. Taking into account the SA of the DMIOLs (SV25T0: $-0.20 \mu \mathrm{m}$; ZKB00: $-0.27 \mu \mathrm{m})$ the maximum remnant SA would be only $+0.07 \mu \mathrm{m}$ in the case of the SV25T0. Because the maximum pupil diameter used in this work was $4.5 \mathrm{~mm}$, one would expect even smaller remnant SA values, ${ }^{45}$ hence, differences in the optical performance of the two DMIOLs associated with differences in the SA compensation have been neglected. Finally, although the ISO standard has established the VIS wavelength range of $546 \pm 10 \mathrm{~nm}$ for IOL testing 25 it is worth remarking that a single wavelength cannot fully represent the optical performance of the human eye under white (or in general polychromatic) light. ${ }^{12,46,47}$

Overall, the discussed results raise concerns about the use of NIR light-based clinical instruments such as aberrometers and double-pass systems to correctly asses the optical quality at far and near distances of patients with DMIOL implants. This may help clinicians to better understand the results obtained when applying aberrometers and double pass systems for the assessment of eyes implanted with these lenses and alert people to a misleading use of such results.

\section{Acknowledgments}

Supported by project DPI2013-43220-R from the Spanish Ministerio de Economía y Competitividad y Fondos FEDER.

Disclosure: F. Vega, None; M.S. Millán, None; N. Vila-Terricabras, None; F. Alba-Bueno, None

\section{References}

1. Artigas JM, Menezo JL, Peris C, Felipe A, Díaz-Llopis M. Image quality with multifocal intraocular lenses and the effect of pupil size: comparison of refractive and hybrid refractivediffractive designs. J Cataract Refract Surg. 2007;33:21112117.

2. Pedrotti E, Mastropasqua R, Passilongo M, Parisi G, Marchesoni I, Marchini G. Comparison of two multifocal intraocular lens designs that differ only in near add. $J$ Refract Surg. 2014;30: 754-760.

3. Wang M, Corpuz CCC, Fujiwara M, Tomita M. Visual and optical performances of multifocal intraocular lenses with three different near additions: 6-month follow-up. Open Ophthalmol J. 2015;9:1-7.

4. Moreno LJ, Piñero DP, Alió JL, Fimia A, Plaza AB. Double-pass system analysis of the visual outcomes and optical performance of an apodized diffractive multifocal intraocular lens. $J$ Cataract Refract Surg. 2010;36(12);2048-2055.

5. Hwang HS, Shin HY, Joo C-K. Double-Pass System (Optical Quality Analysis System) for Analysis of the Multifocal Function of a Diffractive Multifocal Intraocular Lens (Acrysof ReSTOR ${ }^{\circledR}$ ) Compared to a Monofocal Intraocular Lens (Acrysof $\mathrm{IQ}^{\circledR}$ ). J Opt Soc Korea. 2014;18:110-117.

6. Lee H, Lee K, Ahn JM, Kim EK, Sgrignoli B, Kim TI. Evaluation of optical quality parameters and ocular aberrations in multifocal intraocular lens implanted eyes. Yonsei Med J. 2014;55:1413-1420.

7. Pérez-Merino P, Dorronsoro C, Llorente L, Durán S, JiménezAlfaro I, Marcos S. In vivo chromatic aberration in eyes implanted with intraocular lenses. Invest Ophthalmol Vis Sci. 2013;54:2654-2661.

8. Vinas M, Dorronsoro C, Cortes D, Pascual D, Marcos S. Longitudinal chromatic aberration of the human eye in the visible and near infrared from wavefront sensing, double-pass and psychophysics. Biomed Opt Express. 2015;23:513-522. 
9. Thibos LN, Bradley A, Applegate RA. Accuracy and precision of objective refraction from wavefront aberrations. J Vis. 2004; 4:329-351.

10. Fernández E, Unterhuber A, Prieto P, Hermann B, Drexler W, Artal P. Ocular aberrations as a function of wavelength in the near infrared measured with a femtosecond laser. Opt Express. 2005;13:400-409.

11. López-Gil N, Artal P. Comparison of double-pass estimates of the retinal-image quality obtained with green and near-infrared light. J Opt Soc Am A Opt Image Sci Vis. 1997;14:961-971.

12. Ravikumar S, Bradley A, Thibos LN. Chromatic aberration and polychromatic image quality with diffractive multifocal intraocular lenses. J Cataract Refract Surg. 2014;40:11921204.

13. Castignoles F, Flury M, Lepine T. Comparison of the efficiency, MTF and chromatic properties of four diffractive bifocal intraocular lens designs. Opt Express. 2010;18:5245-5256.

14. Alba-Bueno F, Vega F, Millán MS. Halos and multifocal intraocular lenses: Origin and interpretation. Arch la Soc Española Oftalmol (English Ed. 2014;89:397-404.

15. Vega F, Alba-Bueno F, Millán MS, Varón C, Gil MA, Buil JA. Halo and Through-Focus Performance of Four Diffractive Multifocal Intraocular Lenses. Investig Opthalmology Vis Sci. 2015; 56(6);3967.

16. Portney V. Light distribution in diffractive multifocal optics and its optimization. J Cataract Refract Surg. 2011;37:20532059.

17. Turunen J, Wyrovski F. Diffractive Optics for Industrial and Comercial Applications. Berline: Wiley Akademie Verlag;1998.

18. Gatinel D. Double pass-technique limitations for evaluation of optical performance after diffractive IOL implantation. $J$ Cataract Refract Surg. 2011;37:621-622 [author reply 622].

19. Gatinel D, Houbrechts Y. Comparison of bifocal and trifocal diffractive and refractive intraocular lenses using an optical bench. J Cataract Refract Surg. 2013;39:1093-1099.

20. Kim MJ, Zheleznyak L, Macrae S, Tchah H, Yoon G. Objective evaluation of through-focus optical performance of presbyopia-correcting intraocular lenses using an optical bench system. J Cataract Refract Surg. 2011;37:1305-1312.

21. Gundersen KG, Potvin R. Comparative visual performance with monofocal and multifocal intraocular lenses. Clin Opbthalmol. 2013;7:1979-1985.

22. Vega F, Alba-Bueno F, Millán MS. Energy distribution between distance and near images in apodized diffractive multifocal intraocular lenses. Invest Ophthalmol Vis Sci. 2011;52(8); 5695-5701.

23. Vega F, Alba-Bueno F, Millan MS. Energy efficiency of a new trifocal intraocular lens. J Eur Opt Soc Rapid Publ. 2014;9: 14002. doi:10.2971/jeos.2014.14002.

24. International Organization for Standardization. ISO 11979-2: Ophthalmic Implants-Intraocular Lenses-Part 2: Optical Properties and Test Methods. Geneva; ISO;1999.

25. International Organization for Standardization. ISO 11979-9: Ophthalmic Implants-Intraocular Lenses-Part 9: Multifocal Intraocular Lenses. Geneva; ISO;2006.

26. Guirao A, Redondo M, Artal P. Optical aberrations of the human cornea as a function of age. J Opt Soc Am A Opt Image Sci Vis. 2000;17:1697-1702.

27. Wang L, Dai E, Koch DD, Nathoo A. Optical aberrations of the human anterior cornea. J Cataract Refract Surg. 2003;29: 1514-1521.
28. Vega F, Millán MS, Wells B. Spherical lens versus aspheric artificial cornea for intraocular lens testing. Opt Lett. 2010;35: 1539-1541.

29. Norrby S, Piers P, Campbell C, van der Mooren M. Model eyes for evaluation of intraocular lenses. Appl Opt. 2007;46:65956605.

30. Limon O, Zalevsky Z. Ophthalmic halo reduced lenses design. Opt Commun. 2015;342:253-258.

31. Meyers M, Albrecht R. Technique to eliminate scattered light in diffractive optical elements. 1998;.

32. Simpson MJ. Diffractive multifocal intraocular lens image quality. Appl Opt. 1992;31:3621-3626.

33. Toto L, Carpineto P, Falconio G, et al. Comparative study of Acrysof ReSTOR multifocal intraocular lenses $+4.00 \mathrm{D}$ and +3.00 D: visual performance and wavefront error. Clin Exp Optom. 2013;96:295-302.

34. Castillo-Gómez A, Carmona-González D, Martínez-de-la-Casa JM, Palomino-Bautista C, García-Feijoo J. Evaluation of image quality after implantation of 2 diffractive multifocal intraocular lens models. J Cataract Refract Surg. 2009;35:1244-1250.

35. Charman WN, Montes-Mico R, Radhakrishnan H. Problems in the measurement of wavefront aberration for eyes implanted with diffractive bifocal and multifocal intraocular lenses. $J$ Refract Surg. 2007;24:280-286.

36. Schwiegerling J, DeHoog E. Problems testing diffractive intraocular lenses with Shack-Hartmann sensors. Appl Opt. 2010;49:D62-D68.

37. Castignoles F, Lepine T, Chavel P, Cohen G. Shack-Hartmann multiple spots with diffractive lenses. Opt Lett. 2011;36:14221424.

38. Mojzis P, Peña-García P, Liehneova I, Ziak P, Alió JL. Outcomes of a new diffractive trifocal intraocular lens. J Cataract Refract Surg. 2014;40:60-69.

39. Voskresenskaya A, Pozdeyeva N, Pashtaev N, Batkov Y, Treushnicov V, Cherednik V. Initial results of trifocal diffractive IOL implantation. Graefes Arch Clin Exp Ophthalmol. 2010; 248:1299-1306

40. Pieh S. Halo size under distance and near conditions in refractive multifocal intraocular lenses. $\mathrm{Br} \mathrm{J}$ Ophthalmol. 2001;85:816-821.

41. Faklis D, Morris GM. Spectral properties of multiorder diffractive lenses. Appl Opt. 1995;34:2462-2468.

42. Pieh S, Fiala W, Malz A, Stork W. In vitro strehl ratios with spherical, aberration-free, average, and customized spherical aberration-correcting intraocular lenses. Invest Ophthalmol Vis Sci. 2009;50(3);1264-1270.

43. McKelvie J, McArdle B, McGhee C. The influence of tilt, decentration, and pupil size on the higher-order aberration profile of aspheric intraocular lenses. Opbthalmology. 2011; 118:1724-1731.

44. Carson D, Hill WE. Optical bench performance of AcrySof ${ }^{\circledR}$ IQ ReSTOR, AT LISA ${ }^{\circledR}$ tri, and FineVision ${ }^{\circledR}$ intraocular lenses. Clin Opbthalmol. 2014;8:2105-2113.

45. Eom Y, Yoo E, Kang S-Y, Kim H-M, Song J-S Change in efficiency of aspheric intraocular lenses based on pupil diameter. Am J Opbthalmol. 2013;155:492-498.

46. Ravikumar S, Thibos LN, Bradley A. Calculation of retinal image quality for polychromatic light. J Opt Soc Am A Opt Image Sci Vis. 2008;25:2395-2407.

47. Coe C, Bradley A, Thibos LN. Polychromatic Refractive Error from Monochromatic Wavefront Aberrometry. Optom Vis Sci. 2014;91:1167-1174. 
1. Author: the short title "Optical Performance of Diffractive Multifocal Intraocular Lenses" was created for the running header per journal style. Please confirm wording. Copy editor.

2. Author: This article has been lightly edited for grammar, style, and usage. Please compare against your original document and make changes on these pages. Please limit your corrections to substantive changes that affect meaning. If no change is required in response to a question, please write "OK as set" in the margin. Copy editor

3. Author: please clarify Abstract Methods sentence "and variations in the add power"; do you mean, e.g., "positive" power? Copyeditor

4. Author: Abstract Results sentence "whereas the energy efficiency of the near (far) focus decreased (increased)" is unintelligible: do you mean, e.g., "whereas the energy efficiency of the near focus decreased and that of far focus increased"? Copyeditor

5. Author: Carefully check the spelling and order of the author names and check the affiliations for all of the authors.

6. Author: Make sure that ALL funding/financial support is listed in the information following the Acknowledgments.

7. Author: Please supply a brief precis (a 1-2 sentence summary or brief description of your paper) which will be added to the Table of Contents). Copy editor

8. Author: In "Additionally, this," references 22 and 23 appear to refer to "Liou-Brenan and Holladay"; correct? Since there are no authors by those names listed in the present article, please cite the name of the author that appears in your reference list, or if not, supply a complete reference(s) for Liou-Brenan and Holladay. Copyeditor

9. Author: In Tables 1 and 2 - and at first mention in text - please specify what dimension is being referred to as "pupil size" (e.g., diameter?). Also in Tables 1 and 2, please state whether values are, e.g., "mean \pm SD" percentages. Copyeditor 